\title{
Melodic Emotional Expression Increases Ease of Talking to Spoken Dialog Agents
}

\author{
Tomomi Takahashi \\ Kyoto Institute of Technology \\ d9821003@edu.kit.ac.jp \\ Kenichiro Kobayashi \\ TIS Inc. \\ kenichiro.kobayashi@stockmark.co.jp
}

\author{
Kazuaki Tanaka \\ Kyoto Institute of Technology \\ k_tanaka@kit.ac.jp \\ Natsuki Oka \\ Kyoto Institute of Technology \\ nat@kit.ac.jp
}

\begin{abstract}
Many people, especially Japanese, are embarrassed to converse with agents such as virtual assistants, probably due to a low social presence, which refers to the degree to which one perceives the human-like properties of an agent. We assumed that poor emotional expressions of agents may impair their human-likeness. In this study, we proposed melodic emotional expression (MEE), which is a new auditory emotional expression for spoken dialog agents. We added background music (BGM) and sound effects as MEE to synthetic voices and conducted experiments to investigate the effects. First, we found that adding MEE to the flat synthetic voice could convey emotions as intended. We also found that when positive emotions were expressed by MEE, it made the agent more humanlike and easier to talk to. Furthermore, we achieved these effects when MEE was added to an emotional synthetic voice. These effects were particularly noticeable with the BGM. We further attempted automatic BGM generation, which is necessary for the practical application of MEE. Listeners accurately categorized the BGM generated by the prototype system into four types of emotions: joy, angry, sad, and relaxed.
\end{abstract}

\section{CCS CONCEPTS}

- Human-centered computing $\rightarrow$ Human computer interaction (HCI).

\section{KEYWORDS}

Spoken dialog agent, Emotional expression, Background music, Sound effect

\section{ACM Reference Format:}

Tomomi Takahashi, Kazuaki Tanaka, Kenichiro Kobayashi, and Natsuki Oka. 2021. Melodic Emotional Expression Increases Ease of Talking to Spoken Dialog Agents. In Proceedings of the 9th International Conference on HumanAgent Interaction (HAI '21), November 09-11, 2021, Virtual Event, fapan. ACM, New York, NY, USA, 9 pages. https://doi.org/10.1145/3472307.3484180

Permission to make digital or hard copies of all or part of this work for personal or classroom use is granted without fee provided that copies are not made or distributed for profit or commercial advantage and that copies bear this notice and the full citation on the first page. Copyrights for components of this work owned by others than ACM must be honored. Abstracting with credit is permitted. To copy otherwise, or republish, to post on servers or to redistribute to lists, requires prior specific permission and/or a fee. Request permissions from permissions@acm.org.

HAI '21, November 09-11, 2021, Virtual Event, Japan

(C) 2021 Association for Computing Machinery.

ACM ISBN 978-1-4503-8620-3/21/11 ..\$15.00

https://doi.org/10.1145/3472307.3484180

\section{INTRODUCTION}

\subsection{Spoken Dialog Agents}

With the recent progress in AI, human-agent interactions have become common in our daily lives. An increasing number of agents, such as smart speakers for operating home appliances and voice assistants installed in smartphones and cars, are supporting users by utilizing simple voice operations. However, several individuals feel uncomfortable interacting with agents; according to a survey involving 1000 men and women aged 15-69 years in 2017 [1], more than $70 \%$ of people felt uncomfortable with voice control. In addition, in a survey including 1707 men and women aged 18-75 years in 2017 [2], 32\% of individuals were aware of voice assistants, but only $10 \%$ used them. Furthermore, in a survey in 2018 involving 10,000 men and women aged 15-69 years [3], resistance to voice control was cited as a reason for not owning a smart speaker, with a significantly low ownership rate of $5.9 \%$ despite the $76.1 \%$ awareness of smart speakers.

\subsection{Social Presence of Agents}

The resistance to talk to a virtual agent may be owing to perception that the agent is not a creature but an object. It is difficult for agents to be socially interacted with [4][5] because of the low social presence of the agent. Social presence is originally defined as the degree to which the person perceives the presence of the agent through their interaction via a media [6]; however, in the research field regarding the current human-agent interaction (HAI) and human-robot interaction (HRI), social presence is not limited to remote communication and indicates the degree to which a person perceives a human-like nature from an agent [7]. Social presence can be fundamentally improved by increasing the human-likeness of an agent. Therefore, previous studies have focused on making agents more human-like in appearance and behavior or presenting them as physical entities (robots) [8][9][10][11][12][13].

\subsection{Poor Emotional Expressions of Spoken Dialog Agents}

We focused on the emotional expressions of agents to improve their human-likeness. Human beings express emotions and recognize others' emotions through facial expressions and attitudes. However, most existing spoken dialog agents use synthesized speech that is flatter than that of humans and, thus, have fewer emotional expressions. This makes it difficult to convey the agent's emotions; this may reduce the human-likeness and ease of communication. 
Spoken dialog agents, such as smart speakers, are advantageous because they can support users during tasks such as driving and cooking solely through voice interactions. Therefore, it is not appropriate to use visual emotional expressions such as facial expressions, and it is necessary to use auditory information to convey emotions and make people feel the human-like interaction. A situation where the agent expresses emotions through an emotionally synthetic voice may be suitable. The voice assistant named Alexa implements a synthetic voice, such as happy or sad. However, we consider this insufficient for making the synthetic voice sound similar to a human-like emotional tone of voice.

According to Merabian's law [14], the influence of facial expressions (visual), emotional tone of voice (auditory), and speech content (verbal) on judging the emotions of others is $55 \%, 38 \%$, and $7 \%$, respectively. This finding suggests that in the absence of visual information, which influences emotional judgments the most, even if a human-like tone of voice can be reproduced, it may not be an adequate expression of emotion for a spoken dialog agent. This is of greater concern when flat synthetic speech is used. Moreover, creating an emotional synthetic voice that is comparable to the human voice is complex using the available technology. Therefore, the objective of this study is to add melodies to a synthetic voice for a new auditory emotional expression, thereby compensating for the lack of visual emotional expression and the insufficient synthetic voice in an environment where visual information is unavailable.

\subsection{Adding Emotional Expressions to Synthetic Voice through Melody}

In this study, we refer to the proposed method of adding a melody corresponding to the agent's emotions to the synthetic speech as melodic emotional expression (MEE). It has long been stated that music is the language of emotion [15], and the close relationship between music and emotions [16] appears to be intuitively understandable. When listening to music, there are two types of emotional experiences: emotions evoked by the music (e.g. uplifting the listener's own emotions) and emotional impressions of the music (e.g. perception of the music to be uplifting) [17]. Studies regarding singing have also shown that speech and singing voices that express the same emotions have similar acoustical features [18] Therefore, in this study, these properties of music were considered to provide emotion to synthetic speech.

Music is also used as a background and for sound effects (SE) in movies and animations. According to the book describing the role of music in films [19], music is used to convey not only the impression of a scene, but also the emotional state of a character that is not expressed by speech or action to the audience. Thus, we expected that music would be effective in conveying the agents' emotions. In this study, we investigated whether MEE can adequately convey emotions despite the use of flat synthetic speech and whether it can enhance the effect of conveying emotions in emotional synthetic speech.

\subsection{Research Questions}

This study is based on the following three problems.

Research question 1: To investigate the effects of MEE on conveying an emotion.
We examine whether MEE can be adequately conveyed in a speech-only dialog, despite using a flat synthetic speech. In addition, emotional synthetic speech generally appears to be effective in conveying emotions; therefore, we examine whether the effect is enhanced by MEE.

Research question 2: To investigate the effects of MEE on human-like properties and feeling the ease of communication.

We examine whether MEE enhances the agents' human-like properties and ease of talking by enhancing the effect of conveying emotions.

Research question 3: To investigate the types of auditory information that can be used to express emotions to a synthetic voice.

As previously indicated, background music (BGM) and SE may be effective as MEE. Thus, we investigate which of the two types of MEE (BGM and SE) is more effective in conveying emotions, human-like properties, and ease of talking.

\section{RELATED WORKS}

Methods to enhance the social presence of agents and robots have long been studied for HAI and HRI. Several studies have implemented a human-like appearance [8][9][10] and behaviors [9][10][11][12][13] as a basic approach to enhance their social presence. Furthermore, the use of agents with physical entities, i.e., robots, has also been found to be effective in reinforcing their social presence [20], and Robots are more socially accessible than agents on screen [7] and make them more human-like [21]. In addition, the presence of robots in the same room with a participant has been reported to improve task performance [22], suppress fraud [23], that is, the same effects as having a person in the same room were indicated.

Previous studies regarding the methods to make the agents visually more human-like have focused on emotional expressions [10][11][12][13]. For example, a robot has been shown to build a better relationship with humans by separately modeling its facial expressions around the eyes and mouth and allowing it to express expressions such as a bitter smile called Non-Duchenne Smile [10], as well as an expressive robot that reduced boys' personal space [12]. However, it is unclear whether agents with visual emotional expressions can make people feel that agents are human-like, despite using a flat synthetic speech. In addition, as indicated in section 1.3, an emotional expression method that can be applied to agents without visual interactions is needed. In this study, we focused on how agents speaking in synthetic speech should express emotions and investigated the effectiveness of MEE as our proposed method.

There are some studies that aim to express agents' emotions or intentions by using melodies [24][25][26]. A study suggested that music, which were composed and analyzed based on musical factors to express four robot feelings (Happy, Sad, Fear, and Dislike), conveyed stronger emotions than robot's facial expressions and could maximize emotions when the composed music combined with robot's facial expressions [24]. Statistical analysis is expected to increase the reliability of the result. To effectively communicate a robot's affective and internal state, another study proposed an approach for personalizing non-verbal sounds in real-time based on an evolution strategy and human comparative feedback [25]. Furthermore, it is known that a doll, which enable musical expression 
as a communication channel, have a positive effect on conversations between people [26]. These studies suggest that the usefulness of adopting music as a means of expressing the emotions and intentions of agents. However, it is not clear whether the emotional melodies superimposed on synthetic speech can complement or enhance emotions expressed by synthetic speech. It has also not been investigated how the emotional melodies affects the agent's human-likeness and ease of talking.

The following is a study in which the agents used synthetic speech. In the recommendation task, presenting only synthetic speech did not improve the agents' social presence compared with presenting text; however, presenting animation in addition to synthetic speech improved their social presence [27]. In addition, when the pedagogical agent was presented visually, it was reported that using a human voice rather than synthetic speech motivated the user [28] and improved the learning effect [29]. From these findings, it is difficult to create a high social presence by using only synthetic speech, and the effectiveness of visually presenting agents can be observed.

We present examples of studies that successfully enhanced the social presence using only synthetic speech without visual expressions by agents. First, there is a study that represents the presence of multiple agents by reading multiple review articles with multiple types of synthesized speech with different parameters such as pitch and speech speed, which enhances the agents' social presence compared to a single synthesized speech [30]. Then, the same authors also demonstrated that creating introverted/diplomatic synthesized voices and matching them to the introversion/diplomacy of the text enhances agents' social presence [31]. However, as indicated in section 1.3, synthetic speech alone may not be sufficient to convey emotions. Because our proposed method can be used in conjunction with the previous studies, we believe that this may compensate for the lack of visual emotional expressions in the previous studies.

Previous studies have shown that if the performance of an agent is below what the user expects from the agent's appearance and other aspects of the agent's performance as perceived through an actual interaction, it leads to a bad impression (adaptation gap [32]). The same authors proposed a method of adding beeps to the synthesized voice with the aim of conveying the confidence of the agent's decision to the user by the minimum representation necessary to prevent overestimating the agent's performance [33]. Although this method is similar to the proposed MEE method, our study aims to compensate for the lack of visual emotional expressions and focuses on enhancing the agent's human-like properties and to maximize ease of communication.

\section{MELODIC EMOTIONAL EXPRESSION}

We present an experiment to verify the effectiveness of our proposed MEE method. In the experiment, participants evaluated the impression of both synthetic voice alone and a synthetic voice that was added MEE. Because prior impressions of smart speaker voices may vary among participants, all participants listened to a synthetic voice without an added MEE to control the baseline. We prepared two types of synthetic voices, flat and emotional, to investigate whether the proposed method works as an emotional expression when using a flat synthetic voice, and whether it can

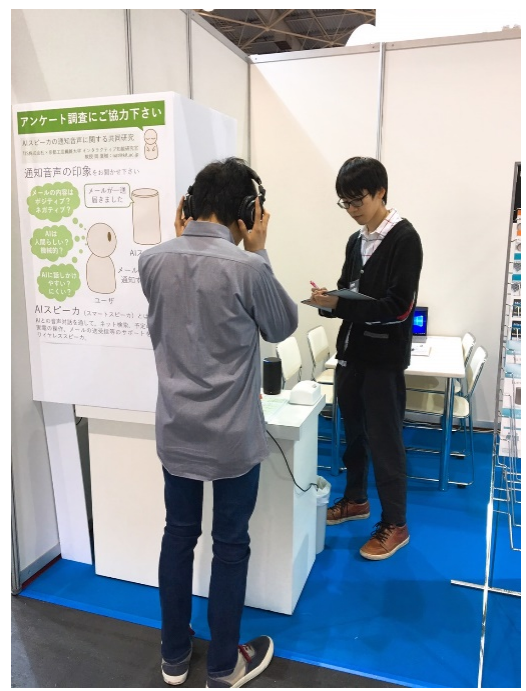

Figure 1: Experimental environment.

enhance the emotions expressed by the synthetic voice when using an emotional synthetic voice. We added MEE to these synthetic voices. MEE adopts melody as the auditory information to add to the synthetic voice; however, it is desirable to investigate what type of melody is effective. Therefore, we prepared the following two types of MEE: BGM and SE. In addition, the following two types of emotions were to be expressed: positive and negative. Hence, there were 8 types of synthetic voices with emotional expressions ( 2 types of synthetic voices, 2 types of melodies, and 2 types of emotions), and each participant experienced one of them.

This experiment was conducted at a company exhibition to evaluate by individuals of different ages, similar to the survey on voice assistants described in section 1 . As a result, 80 people in their $20 \mathrm{~s}$ and $60 \mathrm{~s}$ (60 men and 20 women) participated in the experiment.

\subsection{Procedure}

In the experiment, we simulated a situation where the smart speaker notified participants of receiving an email. The experiment was conducted in the environment shown in Figure 1

First, as a prerequisite for smart speakers, we explained that they are wireless speakers that support internet searching, schedule management, home appliance operation, email sending and receiving, etc., through voice interactions with the virtual assistant. Next, we pointed to a smart speaker in front of the participants and provided the following instructions.

1. This is the scene for notifying participants of receiving an email.

2. The virtual assistant notifies according to the content of the email.

3. Participants are asked to listen to two notification voices.

4. After listening to the audio notifications, participants are asked to evaluate the impression (positive/negative) of the contents in the email, human-like properties, and ease of talking to the virtual assistant. 
Participants listened to two types of notification messages for "An email is received," one with only a synthetic voice, and the other with MEE, and answered a questionnaire. To evaluate the maximum number of individuals at the exhibition, we decided to use a short notification voice. The two types of notifications were counterbalanced by alternating the order of playing for each participant. To prevent the noise of the exhibition from making it difficult for participants to listen to the notifications, they wore headphones and listened from the smart speakers.

Finally, we conducted interviews regarding the reasons for the responses to the questionnaire.

\subsection{Adding MEE to Synthetic Voice}

This section presents the methods for adding MEE (BGM/SE) to a synthetic voice. The synthetic voice was generated by a software ${ }^{1}$ that allows not only a flat synthetic voice, but also an emotional synthetic voice such as happy or sad; the message that was read aloud was "An email was received" under all conditions. For the flat voice, all emotional parameters were set to zero, and for positive/negative voices, the happy/sad parameters were set to the maximum. The duration of the synthetic voice was $1.9 \mathrm{~s}, 1.7 \mathrm{~s}$, and $2.1 \mathrm{~s}$ for the flat, positive, and negative voices, respectively.

BGMs and SEs are free sound sources ${ }^{2}$. Ten laboratory members listened to the selected BGMs and SEs and were confirmed to provide all the impressions, such as positive and negative, as intended.

The first $6.0 \mathrm{~s}$ of both positive and negative original BGMs were used in our experiment. The BGM started to play $1.3 \mathrm{~s}$ before the synthetic voice and started to fade away $1.3 \mathrm{~s}$ after the synthetic voice finished playing.

The duration of the SE was $2.5 \mathrm{~s}$ for both positive and negative SEs, and it started to play $1.3 \mathrm{~s}$ before the synthetic voice, as well as the BGM, started to play.

When the BGMs and SEs were superimposed on the synthetic voice, they were adjusted to be approximately $10 \mathrm{~dB}$ lower for the BGMs and approximately $5 \mathrm{~dB}$ lower for the SEs than the synthesized voice, based on the maximum sound pressure to prevent the synthesized voice from being difficult to hear.

\subsection{Conditions}

To investigate the effects of adding MEE to a flat and emotional synthetic voice, the conditions were based on the following two factors.

MEE factor (within-participant factor, adding/not adding MEE): This factor has two levels, which consists of a synthetic voice alone and a synthesized voice with MEE.

\footnotetext{
${ }^{1}$ AHS Co. Ltd., VOICEROID2 Yuzuki Yukari, https://www.ahsoft.com/voiceroid/yukar (Retrieved July 8, 2021)

${ }^{2}$ positive BGM: PeriTune, "Positive 5", https://peritune.com/ (Retrieved September 11 2021)

negative BGM: Music Atelier Amacha, "Ame no prelude", https://amachamusic chagasi.com/ (Retrieved September 11, 2021)

positive SE: OtoLogic, "Short bridge", https://otologic.jp/ (Retrieved September 11, 2021)

negative SE: MaouDamashii, "One point 29", https://maou.audio/ (Retrieved September 11, 2021)
}

Synthetic voice factor (between-participant factor, flat/emotional): This factor has two levels of the synthetic voice, which are flat and emotional.

Because the MEE factor was within-participants factor, the participants may have been biased if they found the experimenters' intention to verify the effect of adding MEE, and may have evaluated the notification with MEE higher. Therefore, we interviewed the reasons for the responses to the questionnaire to ensure that the respondents did not answer without reason or without understanding the questionnaire items correctly due to such bias. The evaluation scores regarding human-like properties and ease of talking would be high regardless of the type of MEE or emotion, if such a bias had existed. However, as indicated later, such results were not obtained (section 3.5), and the participants provided convincing reasons for their responses in the interviews; therefore, we believe that the bias, if it existed, was negligible.

We describe the assignment of participants and the number of data collected. As shown in Table 1, for one type of MEE, 10 participants were assigned to each level of the synthetic voice factor, that is, 20 people were assigned to the experiment at two levels. Additionally, because the MEE factor had two levels of the within-participants factor (none and adding MEE), two data points were collected per participant. In other words, 40 data points were collected. There were two types of MEE (BGM and SE) and two types of emotions (positive and negative); thus a total of 160 data points were collected for 80 participants.

\subsection{Questionnaire}

All questionnaire items were evaluated using a 7-point semantic differential method, and we asked the participants to answer each of the first and second notifications.

1. What type of email did you feel you received? [Negative Email - Positive Email]

2. How did you feel about the virtual assistant that notified you?

2-3. [mechanical - human-like]

2-4. [difficult to talk to - easy to talk to]

\subsection{Results}

The left side was scored as 1 and the right side as 7 in the adjective pairs of the questionnaire. For each of the BGMs and SEs, a twofactor ANOVA and multiple comparisons were conducted using the TukeyHSD method. The results are shown in Figure 2. The graph presents the mean values of the evaluation, where the error bar indicates the standard error.

3.5.1 Positive MEE. The results of adding the BGM as MEE are shown in the upper left corner in Figure 2. The main effects of the MEE factor were found in all items as follows: email impression $(F(1,18)=100.660, p<.001)$, human-likeness $(F(1,18)=39.710, p<$ $.001)$, and ease of talking $(F(1,18)=25.462, p<.001)$. In the item of impression of the email, the main effect of the synthetic voice factor also had a significant tendency $(F(1,18)=3.176, p=.092)$. Hence, we found that adding the BGM as MEE improved the ease of conveying emotions, human-likeness, and ease of communication, whether the synthetic voice was flat or emotional. 
Table 1: Conditions, levels, and the number of participants.

\begin{tabular}{|l|c|c|}
\hline \multicolumn{1}{|c|}{$\begin{array}{c}\text { Per type of emotions (positive / negative) } \\
\text { per type of MEE (BGM / SE) }\end{array}$} & \multicolumn{2}{c|}{$\begin{array}{c}\text { MEE factor } \\
\text { (Within-participants) }\end{array}$} \\
\cline { 2 - 3 } $\begin{array}{l}\text { Synthetic voice factor } \\
\text { (Between-participant) }\end{array}$ & Flat & None \\
\cline { 2 - 3 } & Emotional & 10 participants \\
\hline
\end{tabular}

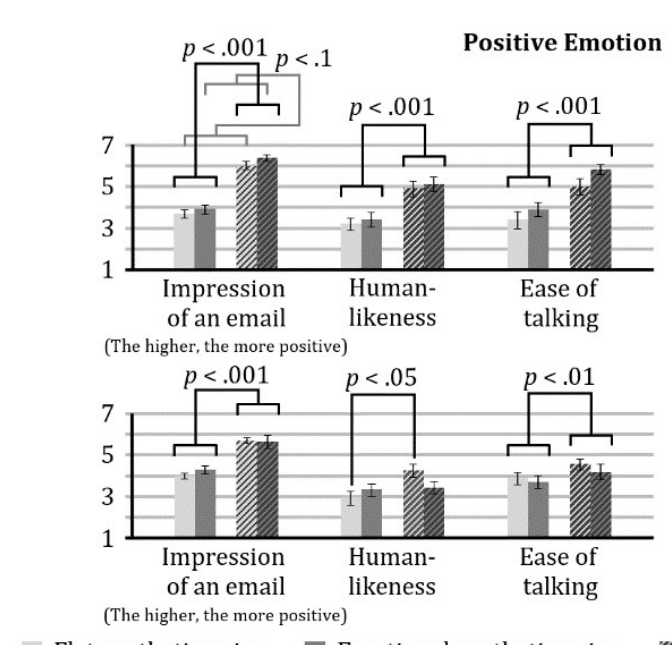

Flat synthetic voice

Emotional synthetic voice

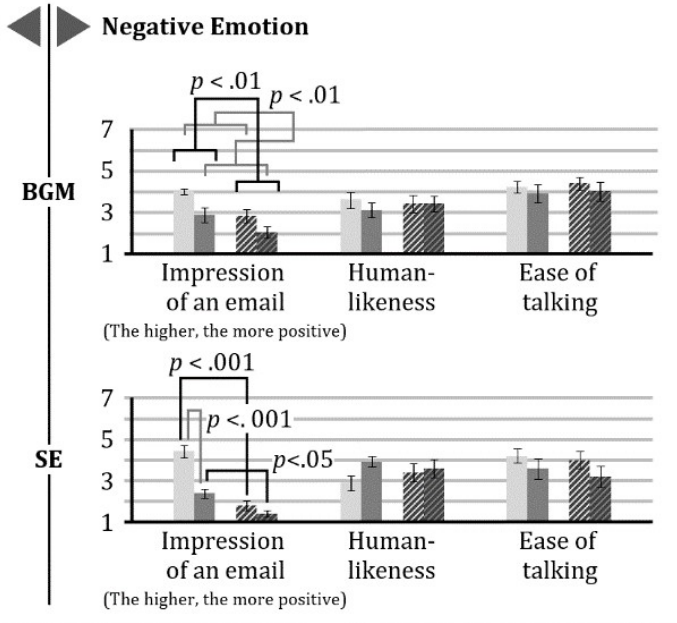

MEE \& flat synthetic voice
MEE \& emotional synthetic voice

Figure 2: Results of the questionnaire

The lower left corner in Figure 2 presents the results for adding the SE as MEE. The main effects of the MEE factor were found in two items as follows: email impression $(F(1,18)=59.648, p<.001)$ and ease of talking to $(F(1,18)=5.345, p<.01)$. Additionally, because the interaction of human-likeness had a significant tendency $(F(1$, $18)=3.927, p=.063)$, multiple comparisons revealed that adding the SE as MEE significantly improved the human-likeness only when the synthetic voice was flat. Therefore, we found that adding the SE as MEE improved the ease of conveying emotions and ease of communication, whether the synthetic voice was flat or emotional. In addition, when the synthetic voice was flat, adding the SE as MEE improved the human-likeness.

3.5.2 Negative MEE. The upper right corner in Figure 2 presents the results for adding the BGM as MEE. The main effects of both the MEE factor $(F(1,18)=13.926, p<.01)$ and synthetic voice factor $(F(1,18)=8.901, p<.01)$ were found in the email impression. Hence, it was found that ease of conveying emotions was improved by the two following methods: adding BGM as MEE whether the synthetic voice was flat or emotional, and using an emotional synthetic voice whether the BGM was added as MEE.

The results for adding the SE as MEE are shown in the lower corner in Figure 2. The interaction of the email impression was found $(F(1,18)=8.727, p<.01)$, and multiple comparisons revealed that adding the SE significantly improved the ease of conveying emotions whether the synthetic voice was flat $(p<.001)$ or emotional $(p<.05)$. Additionally, in the case of notification without the SE, emotional synthetic voice also significantly improved the ease of conveying emotions $(p<.001)$. Therefore, it was found that using the SE for emotional expressions improved the ease of conveying emotions whether the synthetic voice was flat or emotional, and using an emotional synthetic voice also improved the ease of conveying emotions.

\subsection{Discussion}

3.6.1 Conveying Emotions by MEE. As a result of the experiment, it was found that adding MEE to a flat synthetic voice conveyed emotions regardless of whether the emotions to be conveyed were positive or negative. In addition, it was shown that emotions were conveyed more easily when adding MEE to an emotional synthetic voice.

Using an emotional synthetic voice made conveying emotions significantly easier only when the emotions were negative. In daily life, people often hear either flat, unemotional, or amiable synthetic voices, such as those implemented in household appliances. In other words, participants may be accustomed to hearing bright synthetic voices. Hence, it might be difficult for them to perceive the positive synthetic voice in our experiment to be positive voices clearly, and it is possible that the positive synthetic voice used in this experiment was perceived as general and neutral. In contrast, because the participants were unfamiliar to the negative synthetic voice, it is likely that the negative emotion could be conveyed despite of using an emotional synthetic voice alone. 
MEE enhanced the ability of a synthetic voice to convey emotions, regardless of the type of emotions. This study suggests that MEE can be used to convey emotions by using auditory information alone in conversations with a spoken dialog agent, such as a smart speaker, despite the unavailability of visual facial expressions.

3.6.2 Improving Human-likeness and Ease of Talking by MEE. Adding MEE improved human-likeness and ease of talking to. According to the interviews with the participants, the reasons for feeling human-like properties and ease of talking were often the same, and it is possible that the human-likeness of smart speakers may be related to the ease of talking to them. In the following, we discuss the results of the experiments based on the opinions obtained in the interviews.

When the BGM was added to the synthetic voice, we obtained opinions where the participants felt that the melody made the synthetic voice sound brighter: for example, "The melody made the voice sound gentler and more human-like," and "I felt the synthetic voice, which added BGM, human-like because it was heard brighter than another." Similarly, when BGM was added to the emotional synthetic voice, participants scored higher on human-likeness and ease of talking to because they felt differences in the emotional synthetic voice, although the emotional synthetic voices were the same between the synthetic voice without BGM and one with BGM, stating, "the voice was not as sharp in the notification with melody," "the voice quality was younger in notification with melody," and "the melody suppresses too much tension in the voice." These opinions suggest that adding BGM to synthetic voices may have the effect of reducing the mechanical impression of synthetic voices and increasing human-likeness and ease of talking to.

However, these effects of BGM were only found when positive emotions were expressed. Even when expressing negative emotions, some participants felt that the addition of BGM changed the synthetic voice, and some increased their scores for human-likeness and ease of talking to, because they stated "I felt like the virtual assistant had emotions so it was human-like." However, some participants lowered those scores because they did not want to be negatively notified in case of negative contents. The idea that negatively notifying participants in a negative situation, where they receive negative content in the email, is not human-like, may have denied the effect which improves human-like and ease of talking to of adding BGM. If so, it is possible that if the situation was not negative for the participants, it may have had the same effect as the positive emotional expression. For example, it may be useful when the agent says statements such as "I missed you for a while." It may also be useful to express encouraging, instead of negative, emotions despite negative email notification tasks.

Similar to BGM, the use of SE was found to improve ease of talking to when positive emotions were expressed. However, for human-likeness, the effect was only found when SE was added to a flat synthetic voice. A few participants increased their scores for human-likeness and ease of talking to because "the sound made the voice seem brighter" and "the sound changes the impression," but the SE was shorter than the BGM, which may have weakened the effect.

It is believed that emotions need to be clearly conveyed to people to make agents feel alive [34], and it indicates that techniques such as making animated characters feel alive with exaggerated emotional expressions [35] may be effective. Expressing character emotions through music also appears to be adopted as a method to have the emotions conveyed to the audience more easily [19]. Exaggerated emotions and emotional expressions by melody are not used in everyday conversations between people, unlike in animations; however, the audience feels these characters are human-like. In this study, MEE with BGMs and SEs also enhanced the humanlike impression by clarifying agent emotions. This study suggests that agents, which is similar to animated characters, may not only mimic human emotional expressions, but also exaggerate them to make them more human-like.

We obtained an opinion indicating that participants were able to be ready to listen to the message of the notification because the BGMs and SEs were played ahead of time, instead of suddenly being notified by a voice. When people talk to each other, they are likely to present an intention to talk because they might miss hearing what they say or be surprised, if they speak abruptly. In addition, to make the animated characters feel alive, there is a known technique to make the audience anticipate what the characters are going to do by anticipation motions so that their emotional states and behaviors can be easily understood [35]. It is possible that BGMs and SEs initiated this type of anticipated motions and contributed to improve human-likeness.

In this study, we conducted an evaluation using a simple task of email notification and a direct questionnaire. To investigate the more detailed effectiveness of the proposed method, we will consider conducting experiments adopting behavioral indicators such as the frequency that users talk to an agent in conversation tasks and multifaceted evaluation indicators such as Godspeed [36].

3.6.3 More Effective Auditory Information as MEE. Both the BGM and SE were effective in enhancing the ability to convey emotions and improved ease of talking to. Especially, the use of the BGM was effective in enhancing the human-likeness of the smart speaker, not only for the flat synthetic voice but also for the emotional synthetic voice.

BGM is also expected to be a MEE suitable for emotional synthetic speech. This is because all the evaluation items in the upper left corner of Figure 2 shows that the MEE \& Emotional Synthetic Voice condition is the highest score among all the conditions. In the future, when emotional synthetic voices are implemented in spoken dialog agents, such as smart speakers, the use of BGMs as emotional expressions will exert synergy with the emotional expressions by synthetic voices and function as the most effective way of expressing emotions.

\section{TOWARDS AUTOMATIC MEE GENERATION}

In this section, we discuss the potential to generate MEE automatically and describe the prototype system (MEE system). We note that this section is simple trial to generate MEE automatically and that applying deeper and more accurate insights about music, art, and music generation [24][25] is our future work.

The BGMs and SEs used in the experiments described in section 3 were selected in advance by the experimenters. However, for implementing MEE in real voice assistants, it is desirable to 


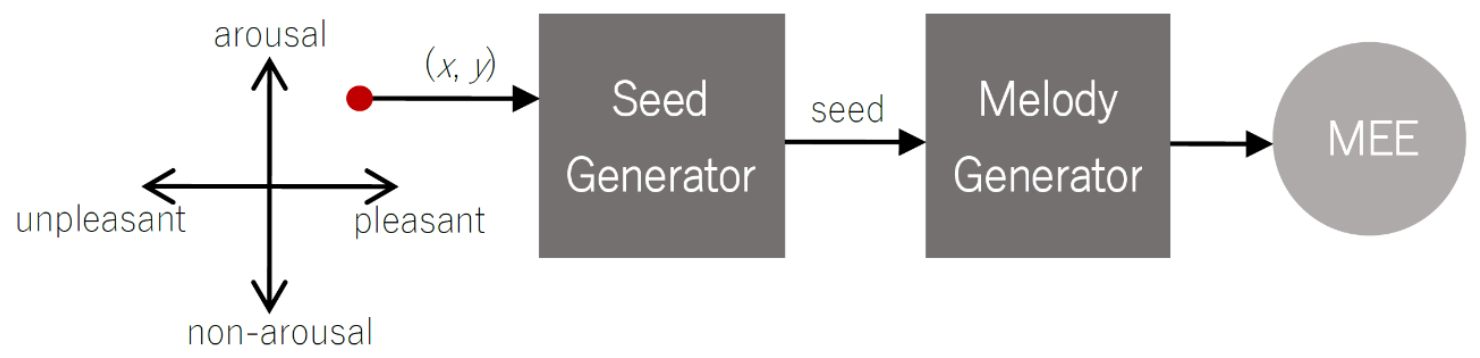

Figure 3: Overview of MEE system

automatically generate the appropriate MEE each time, according to the emotions to be expressed. We hence decided to automate the generation of MEE. In addition, as explained in the previous section, the use of BGM as MEE was expected to have a high effect, and therefore, we adopted BGMs as MEE.

\subsection{Overview}

The flow of generating MEE in the prototype system is shown in Figure 3. First, the emotions to be conveyed were expressed numerically and input to the system. The values were set from 1.0 to -1.0 on the horizontal axis (pleasant-unpleasant, referred to as the valence axis) and vertical axis (arousal-non-arousal, referred to as the arousal axis) of Russell's circumplex model [37]. In this study, we expressed the value of the valence axis as $\mathrm{x}$ and the value of the arousal axis as $y$.

There are several studies regarding models generating music [38][39][40][41][42], and these often adopt the method where the next note is predicted from first few notes that determine the flow of melody by using machine learning methods such as recurrent neural network (RNN); we focused on Magenta [41] as the melody generator. The seed generator generated the seed from the input emotion values by mapping the valence axis to the key and the arousal axis to the length of the note. The seed was input to the melody generator and a melody was generated. The property that a melody generated following the input seed tends to have the same impression as the seed impression is useful for generating MEE. The detailed processing of each part is described as follows.

\subsection{Seed Generator}

In the melody generator, which is presented later, it is necessary to input the first few notes of the melody to be generated, basic length of one chord, and chord progression of the entire melody as the seed. First, the key was determined according to the value of $\mathrm{x}$ : the positive/negative value of $\mathrm{x}$ was corresponded to major/minor keys, and the code was decided based on an author's subjectivity mainly. The first note was the tonic in the scale of the determined key, and the following notes were selected from the scale for the number of notes that one wants to input as the seed. These notes were selected randomly. The longer notes were expected to provide a more relaxed and non-arousal feeling. Hence, it was designed such that when y was negative, a half note was selected with y probability, and when y was positive, an eighth note was selected with y probability; otherwise, it was a quarter note.
Next, the seed generator determined the basic length of each code. In other words, the seed generator specified the number of steps per chord; a larger value indicates a slower melody. This value was determined based on Table 2

Finally, the generator selected one of the chord progressions registered in advance (canon progression, three-chord progression, etc.). If $\mathrm{x}$ was positive, the major code was selected, and if $\mathrm{x}$ was negative, the minor code was selected. Whether the duration of each chord in the selected chord progression should be the basic length of one chord or the length of multiple chords should be determined by the value of the arousal axis, for the same reason as determining the length per note in the first few notes. Therefore, when y was negative, the current chord was continued with a probability of $y$, and when y was positive, the current chord was continued with a probability of 1-y; otherwise, the basic length was set. Because an extended continuation would be unnatural for a melody, we set the upper limit of the continuation to two chords in the prototype. In addition, the melody generator needed this chord progression for the length of the melody to be generated. Therefore, the chord progression was created repeatedly until the desirable duration of the melody to be generated is reached.

\subsection{Melody Generator}

For the melody generator, we used a model called Improv RNN [43], which was developed in the magenta project for art and music generation. The three inputs needed as seeds were the first few notes of the melody, basic length of one chord, and chord progression of the entire melody, as described in the previous section. This model is unique because it allows us to specify a chord progression.

\subsection{Generated Melodies}

We asked several individuals to listen to several melodies generated by the prototype system and got feelings that the melodies generally match with intended emotions in the case of classifying emotions into four types: joy, angry, sad, and relaxed. Conducting a rigorous and detailed evaluation experiment is one of our future tasks. Furthermore, we thought that we should investigate insights about music, art, and music generation and apply in the future to the mapping described in section 4.2 . 
Table 2: Tentative correspondence between y (upper) and the number of steps per chord (lower).

\begin{tabular}{|l|l|l|l|l|}
\hline$<-0.7$ & $<-0.4$ & $<0.4$ & $<0.7$ & $<1.0$ \\
\hline 32 & 16 & 8 & 4 & 2 \\
\hline
\end{tabular}

\section{CONCLUSION}

Most of the current spoken dialog agents use only flat synthetic voices, and a lack of emotional expression may lead to decreased human-likeness and resistance to talk to objects.

Clear emotional expressions such as facial expressions can be used in situations where the agent can be visually presented and interacted with; however, auditory emotional expressions are required in situations where the user needs to be assisted only by voice. We proposed MEE as an auditory emotional expression and conducted an experiment to verify its effectiveness. As a result of adding BGM and SEs as MEE to the synthetic voice and evaluating the impression, MEE was able to convey emotions even with the flat synthetic voice. Despite MEE being added to the emotional synthetic voice, the effect of conveying emotions could be enhanced. In addition, when BGM and SEs were used for positive emotional expressions, the human-likeness of the agent and the ease of talking to could be improved. The effects of BGM as MEE were the most prominent.

In addition, we have started to automatically generate MEE for implementation in real smart speakers and other spoken dialog agents.

The results of this study, which revealed the effect of adding MEE to synthetic voices of spoken dialog agents, will contribute to the fields of HAI/HRI and musical information processing. Furthermore, we hope that the effect of MEE on enhancing emotional expressions by voice will be applied to the interface that mediates the interaction between people and systems, and conveying emotions will be facilitated.

\section{ACKNOWLEDGMENTS}

This work was supported by JSPS KAKENHI Grant Numbers JP19K12081, JP20H05564, JP21J15349.

\section{REFERENCES}

[1] Awareness Survey on Japanese Voice Operations 2017. KDDI CORPORATION. Retrieved July 8, 2021 from http://news.kddi.com/kddi/corporate/newsrelease/ 2017/10/05/2726.html

[2] Global Mobile Consumer Survey 2017. Deloitte. Retrieved July 8, 2021 from https://www2.deloitte.com/jp/ja/pages/technology-media-andtelecommunications/articles/com/mobile-consumer-survey-2017.html

[3] Survey on domestic use of smart speakers. Dentsu Digital Inc. Retrieved July 8 , 2021 from https://www.dentsudigital.co.jp/release/2019/0218-000164/

[4] Min Kyung Lee, Sara Kiesler, Jodi Forlizzi, and Paul Rybski. 2012. Ripple effects of an embedded social agent: a field study of a social robot in the workplace. In Proceedings of the SIGCHI Conference on Human Factors in Computing Systems. ACM, New York, NY, USA, 695-704. https://doi.org/10.1145/2207676.2207776

[5] Kazuaki Tanaka, Naomi Yamashita, Hideyuki Nakanishi, and Hiroshi Ishiguro. 2016. Teleoperated or autonomous?: How to produce a robot operator's pseudo presence in HRI. In Proceedings of the 11th ACM/IEEE International Conference on Human-Robot Interaction. IEEE, Christchurch, NZL, 133-140. https://doi.org/ 10.1109/HRI.2016.7451744

[6] John Short, Ederyn Williams, and Bruce Christie. 1976. The Social Psychology of Telecommunications. Wiley.

[7] Wilma A. Bainbridge, Justin W. Hart, Elizabeth S. Kim, and Brian Scassellat. 2011 The Benefits of Interactions with Physically Present Robots over Video-Displayed Agents. Int J of Soc Robotics 3, 1 (October 2011), 41-52. https://doi.org/10.1007/ s12369-010-0082-7
[8] Nick Yee, Jeremy N Bailenson, and Kathryn Rickertsen. 2007. A meta-analysis of the impact of the inclusion and realism of human-like faces on user experiences in interfaces. In Proceedings of the SIGCHI Conference on Human Factors in Computing Systems. ACM, New York, NY, USA, 1-10. https://doi.org/10.1145/ 1240624.1240626

[9] Abolfazl Zaraki, Daniele Mazzei, Manuel Giuliani, and Danilo De Rossi. 2014. Designing and Evaluating a Social Gaze-Control System for a Humanoid Robot. IEEE Transactions on Human-Machine Systems 44, 2 (April 2014), 157-168. https: //doi.org/10.1109/THMS.2014.2303083.

[10] Naoki Koyama, Kazuaki Tanaka, Kohei Ogawa, and Hiroshi Ishiguro. 2017. Emotional or Social? How to Enhance Human-Robot Social Bonding. In Proceedings of the 5th International Conference on Human Agent Interaction. ACM, New York, NY, USA, 203-211. https://doi.org/10.1145/3125739.3125742

[11] Cynthia Breazeal. 2003. Emotion and sociable humanoid robots. Int. J. Hum.Comput. Stud. 59, 1-2 (July 2003), 119-155. https://doi.org/10.1016/S10715819(03)00018-1

[12] David Cameron, Samuel Fernando, Emily C. Collins, A. Millings, Roger K. Moore, A. Sharkey, V. Evers and T. Prescott. 2015. Presence of Life-Like Robot Expressions Influences Children's Enjoyment of Human-Robot Interactions in the Field. In Proceedings of the 4th International Symposium on New Frontiers in HumanRobot Interaction. 36-41.

[13] André Pereira, Rui Prada, and Ana Paiva. 2014. Improving social presence in human-agent interaction. In Proceedings of the SIGCHI Conference on Human Factors in Computing Systems. ACM, New York, NY, USA, 1449-1458. https: //doi.org/10.1145/2556288.2557180

[14] Albert Mehrabian. 1972. Nonverbal Communication. Aldine Transaction.

[15] Deryck Cooke. 1959. The language of music. Oxford University Press.

[16] Stephen Davies. 1980. The Expression of Emotion in Music. Mind 89, 353 (January 1980), 67-86

[17] Kazuma Mori, Makoto Iwanaga. 2014. Recent progress in music and emotion studies: Psychological response, peripheral nervous system activity, and musicoacoustic features. Japanese Psychological Review 57, 2 (March 2014), 215-234. https://doi.org/10.24602/sjpr.57.2_215 (in Japanese)

[18] Klaus R. Scherer. 1995. Expression of Emotion in Voice and Music. Journal of Voice 9, 3 (September 1995), 235-248. https://doi.org/10.1016/S0892-1997(05)80231-0

[19] Richard Davis. 2010. Complete Guide to Film Scoring: The Art and Business of Writing Music for Movies and TV. Berklee Press.

[20] Kwan Min Lee, YounboJung, Jaywoo Kim, and Sang Ryong Kim. 2006. Are physically embodied social agents better than disembodied social agents?: The effects of physical embodiment and tactile interaction and people's loneliness in human-robot interaction. International Journal of Human-Computer Studies 64, 10 (October 2006), 962-973.

[21] Sara Kiesler, Aaron Powers, Susan R. Fussell, and Cristen Torrey. 2008. Anthropomorphic interactions with a robot and robot-like agent. Social Cognition 26, 2 (April 2008), 169-181. https://doi.org/10.1521/soco.2008.26.2.169

[22] Nina Riether, Frank Hegel, Britta Wrede, and Gernot Horstmann. 2012. Social facilitation with social robots? In Proceedings of the seventh annual ACM/IEEE international conference on Human-Robot Interaction. ACM, New York, NY, USA, 41-48. https://doi.org/10.1145/2157689.2157697

[23] Guy Hoffman, Jodi Forlizzi, Shahar Ayal, Aaron Steinfeld, John Antanitis, Guy Hochman, Eric Hochendoner, and Justin Finkenaur. 2015. Robot Presence and Human Honesty: Experimental Evidence. In Proceedings of the Tenth Annual ACM/IEEE International Conference on Human-Robot Interaction. ACM, New York, NY, USA, 181-188. https://doi.org/10.1145/2696454.2696487

[24] Eun-Sook Jee, Chong Hui Kim, Soon-Young Park, and Kyung-Won Lee. 2007. Composition of Musical Sound Expressing an Emotion of Robot Based on Musical Factors. In Proceedings of the RO-MAN 2007 - The 16th IEEE International Symposium on Robot and Human Interactive Communication. IEEE, Jeju, Korea, 637-641. https://doi.org/10.1109/ROMAN.2007.4415161

[25] Hannes Ritschel, Ilhan Aslan, Silvan Mertes, Andreas Seiderer, and Elisabeth André. 2019. Personalized Synthesis of Intentional and Emotional Non-Verbal Sounds for Social Robots. In Proceedings of the 2019 8th International Conference on Affective Computing and Intelligent Interaction (ACII). IEEE, Cambridge, UK, 1-7. https://doi.org/10.1109/ACII.2019.8925487

[26] Tomoko Yonezawa and Kenji Mase. 2002. Musically expressive doll in face-to-face communication. In Proceedings of the Fourth IEEE International Conference on Multimodal Interfaces. IEEE, Pittsburgh, PA, USA, 417-422. https://doi.org/10. 1109/ICMI.2002.1167031 
[27] Traci J. Hess, Mark Fuller, and Damon E. Campbell. 2009. Designing Interfaces with Social Presence: Using Vividness and Extraversion to Create Social Recommendation Agents. Journal of the Association for Information Systems 10, 12 (December 2009), Article 1. https://doi.org/10.17705/1jais.00216

[28] Yanghee Kim, Amy L. Baylor, and Gabrielle Reed. 2003. The impact of image and voice with pedagogical agents. In Proceedings of the E-Learn 2003-World Conference on E-Learning in Corporate, Government, Healthcare, and Higher Education. AACE, Phoenix, Arizona, USA, 2237-2240.

[29] Robert K. Atkinson, Richard Mayer, and Mary Margaret Merrill. 2005. Fostering social agency in multimedia learning: Examining the impact of an animated agent's voice. Contemporary Educational Psychology 30, 1 (January 2005), 117139. https://doi.org/10.1016/j.cedpsych.2004.07.001

[30] Kwan Min Lee and Clifford Nass. 2004. The multiple source effect and synthesized speech: Doublydisembodied language as a conceptual framework. Human Communication Research 30, 2 (April 2004), 182-207. https://doi.org/10.1111/j.14682958.2004.tb00730.x

[31] Kwan Min Lee and Clifford Nass. 2003. Designing social presence of social actors in human computer interaction. In Proceedings of the SIGCHI Conference on Human Factors in Computing Systems. ACM, New York, NY, USA, 289-296. https://doi.org/10.1145/642611.642662

[32] Takanori Komatsu and Seiji Yamada. 2011. Adaptation gap hypothesis: How differences between users' expected and perceived agent functions affect their subjective impression. Journal of Systemics 9, 1 (February 2011), 67-74.

[33] Takanori Komatsu, Seiji Yamada, Kazuki Kobayashi, Kotaro Funakoshi, and Mikio Nakano. 2010. Artificial subtle expressions: intuitive notification methodology of artifacts. In Proceedings of the SIGCHI Conference on Human Factors in Computing Systems. ACM, New York, NY, USA, 1941-1944. https://doi.org/10. $1145 / 1753326.1753619$
[34] Joseph Bates. 1994. The role of emotion in believable agents. Commun. ACM 37 , 7 (July 1994), 122-125. https://doi.org/10.1145/176789.176803

[35] Frank Thomas and Ollie Johnston. 1981. Disney Animation: The Illusion of Life. Abbeville Press.

[36] Christoph Bartneck, Dana Kulić, Elizabeth Croft, and Susana Zoghbi. 2009. Measurement Instruments for the Anthropomorphism, Animacy, Likeability, Perceived Intelligence, and Perceived Safety of Robots. Int J of Soc Robotics 1 (October 2008), 71-81. https://doi.org/10.1007/s12369-008-0001-3

[37] James A Russell. 1980. A circumplex model of affect. Journal of Personality and Social Psychology 39, 6 (December 1980), 1161-1178. https://doi.org/10.1037/ h0077714

[38] Jesse Engel, Kumar Krishna Agrawal, Shuo Chen, Ishaan Gulrajani, Chris Donahue, and Adam Roberts. 2019. GANSynth: Adversarial Neural Audio Synthesis. arXiv preprint. arXiv: 1902.08710

[39] Li-Chia Yang, Szu-Yu Chou, and Yi-Hsuan Yang. 2017. MidiNet: A Convolutional Generative Adver-sarial Network for Symbolic-domain Music Generation. arXiv preprint. arXiv: 1703.10847

[40] Aaron van den Oord, Sander Dieleman, Heiga Zen, Karen Simonyan, Oriol Vinyals, Alex Graves, Nal Kalchbrenner, Andrew Senior, and Koray Kavukcuoglu. 2016. WaveNet: A Generative Model for Raw Audio. arXiv preprint. arXiv: 1609.03499

[41] Magenta. Google Brain team. Retrieved September 12, 2021 from https://github. com/magenta/magenta

[42] MuseNet. OpenAI. Retrieved September 12, 2021 from https://openai.com/blog/ musenet/

[43] Improv RNN. Google Brain team. Retrieved September 12, 2021 from https: //github.com/magenta/magenta/tree/master/magenta/models/improv_rnn 